\title{
Combined Administration of Recombinant Human Megakaryocyte Growth and Development Factor and Granulocyte Colony-stimulating Factor Enhances Multilineage Hematopoietic Reconstitution in Nonhuman Primates after Radiation- induced Marrow Aplasia
}

\author{
Ann M. Farese, ${ }^{\star \ddagger}$ Pamela Hunt,,$\$$ Lisa B. Grab, ${ }^{\star \ddagger}$ and Thomas J. MacVittie ${ }^{\star \ddagger}$ \\ *Experimental Hematology, Armed Forces Radiobiology Research Institute, Bethesda, Maryland 20889; ${ }^{\ddagger}$ University of Maryland Cancer \\ Center, Baltimore, Maryland 21201; and ${ }^{\S}$ Amgen Inc., Thousand Oaks, California 91320
}

\begin{abstract}
This study compared the therapeutic potential of recombinant, native versus pegylated megakaryocyte growth and development factor (rMGDF and PEG-rMGDF, respectively), as well as that of the combined administration of PEG-rMGDF and r-methionyl human granulocyte colonystimulating factor (r-metHuG-CSF) on hematopoietic reconstitution after $700 \mathrm{cGy},{ }^{60} \mathrm{Co}$ gamma, total body irradiation in nonhuman primates. After total body irradiation, animals received either rMGDF, PEG-rMGDF, r-metHuGCSF, PEG-rMGDF and r-metHuG-CSF, or HSA. Cytokines in all MGDF protocols were administered daily for $18 \mathrm{~d}, \mathrm{HSA}$ and r-metHuG-CSF alone were administered for 21-23 d.

Either rMGDF, PEG-rMGDF, or PEG-rMGDF and r-metHuG-CSF administration significantly diminished the thrombocytopenic duration (platelet count [PLT] $<20,000 /$ $\mu l)$ to $0.25,0$, and $0.5 \mathrm{~d}$, respectively, and the severity of the PLT nadir $(28,000,43,000$, and $30,000 / \mu 1$, respectively) as compared with the controls (12.2 d duration, nadir 4,000/ $\mu \mathrm{l}$ ), and elicited an earlier PLT recovery. Neutrophil regeneration was augmented in all cytokine protocols and combined PEG-rMGDF and r-metHuG-CSF further decreased the duration of neutropenia compared with r-metHuG-CSF alone.

These data demonstrated that the administration of PEGrMGDF significantly induced bone marrow regeneration versus rMGDF, and when combined with r-metHuG-CSF significantly enhanced multilineage hematopoietic recovery with no evidence of lineage competition. (J. Clin. Invest. 1996. 97:2145-2151.) Key words: platelet count • thrombocytopenia $\bullet$ radiation $\bullet$ cytokines $\bullet$ primates
\end{abstract}

\section{Introduction}

Major dose limiting complications of radiotherapy and/or chemotherapy, particularly in the context of multiple cycles of

Address correspondence to Ann M. Farese, M.S., University of Maryland Cancer Center, MSTF-Room G-05, 10 South Pine St., Baltimore, MD 21201. Phone: 410-706-0909; FAX: 410-706-0928.

Received for publication 25 August 1995 and accepted in revised form 27 February 1996.

The Journal of Clinical Investigation

Volume 97, Number 9, May 1996, 2145-2151 cytotoxic therapy, are thrombocytopenia and neutropenia. Granulocyte colony-stimulating factor (G-CSF) reduces the duration of neutropenia in preclinical $(1-6)$ and clinical $(7,8)$ scenarios of myelosuppression. Effective treatment of thrombocytopenia has remained elusive.

Recently the cognate ligand for the c-mpl receptor has been identified, cloned, and produced (9-14). The mpl ligand, also referred to as megakaryocyte growth and development factor (MGDF), ${ }^{1}$ thrombopoietin (Tpo), or megapoietin, has shown a range of in vitro biologic activity that strongly suggests it is a cytokine capable of stimulating both megakaryocytopoiesis and thrombopoiesis (9-20). Early preclinical studies in normal rodents have demonstrated the suspected efficacy of recombinant (r) MGDF $(21,22)$ or Tpo (11-15) to increase the levels of circulating platelets, marrow megakaryocytes, and megakaryocyte (MEG) colony-forming units (CFU) relative to levels noted for IL-3 $(23,24)$, IL-6 (25), leukemia inhibitory factor (26), and IL-11 (27). In addition, administration of rMGDF to normal rhesus monkeys elicited a lineage-specific, sixfold increase in platelet levels 2-3 d after cessation of 10 consecutive days of treatment (28). The efficacy of rMGDF has also been demonstrated in the modulation of platelet nadir and the stimulation of platelet recovery following myelosuppression in rodents (21).

A pegylated (PEG) form of the native molecule (rMGDF) produced by Amgen Inc. exhibits an increased circulating halflife and roughly a 20-fold increase in its in vivo activity in normal mice (22). In addition, Hokom et al. (22) showed that the coadministration of PEG-rMGDF and murine G-CSF increased survival versus control and G-CSF-treated mice in a combined carboplatin-irradiation murine model, as well as enhancing both platelet and neutrophil recovery relative to the single cytokine treatment protocols. The purpose of this study was to compare the relative efficacy of the pegylated versus the nonglycosylated rMGDF and to further elucidate the role of PEG-rMGDF either alone or in combination with G-CSF in the enhancement of hematopoietic reconstitution in a highdose, sublethal, nonhuman primate model of radiation-induced myelosuppression.

1. Abbreviations used in this paper: ANC, absolute neutrophil count; BFU-e, burst-forming unit-erythroid; BM, bone marrow; GEMM, granulocyte erythroid megakaryocyte macrophage; MEG, megakaryocyte; MGDF, megakaryocyte growth and development factor; MNC, mononuclear cells; PEG, pegylated; PLT, platelet count; r-metHu, recombinant methionyl human; TBI, total body irradiation; Tpo, thrombopoietin. 


\section{Methods}

\section{Animals}

Domestic born male rhesus monkeys, Macaca mulatta, mean weight $3.9 \pm 0.2 \mathrm{~kg}$, were housed in individual stainless steel cages in conventional holding rooms at the Armed Forces Radiobiology Research Institute in an animal facility accredited by the American Association for Accreditation of Laboratory Animal Care. Monkeys were provided 10 air changes $/ \mathrm{h}$ of $100 \%$ fresh air, conditioned to $72 \pm 2^{\circ} \mathrm{F}$ with a relative humidity of $50 \pm 20 \%$, and were maintained on a 12 -h light/ dark full spectrum light cycle, with no twilight. Monkeys were provided with commercial primate chow, supplemented with fresh fruit and tap water ad libitum. Research was conducted according to the principles enunciated in the Guide for the Care and Use of Laboratory Animals (29), prepared by the Institute of Laboratory Animal Resources, National Research Council.

\section{Irradiation}

Monkeys placed in a Lucite restraining chair, after a prehabituation period, were bilaterally, total body irradiated (TBI) with ${ }^{60} \mathrm{Co}$ gamma radiation to a total midline dose of $700 \mathrm{cGy}$ at a dose rate of $40 \mathrm{cGy} /$ min. Dosimetry was performed using paired $0.5 \mathrm{~cm}^{3}$ ionization chambers, with calibration factors traceable to the National Institute of Standards and Technology.

\section{Cytokines}

Cytokines were provided by Amgen Inc. Recombinant MGDF was provided as either a nonglycosylated Escherichia coli-derived, truncated molecule (rMGDF) or as this molecule which was modified by derivatization with poly-ethylene glycol (PEG-rMGDF). Both species were purified to homogeneity before use (Amgen Inc.). In vitro, the specific activities of rMGDF and PEG-rMGDF did not differ by $>25 \%$. Specific activities, based on the peptide molecular weight, were determined with a factor-dependent murine cell line transfected with the human c-mpl gene where one unit results in half-maximal growth stimulation (9). Doses of rMGDF $(25 \mu \mathrm{g} / \mathrm{kg} / \mathrm{d})$ and PEGrMGDF $(2.5 \mu \mathrm{g} / \mathrm{kg} / \mathrm{d})$ were chosen based on Amgen Inc. proprietary data indicating that the PEG-rMGDF protein has an extended serum half-life in rodents and nonhuman primates and a significant increase in biological activity such that a 10 -fold decrease in dose would yield an equivalent or better biological activity. Neupogen ${ }^{\circledR}$ (Filgrastim), r-metHuG-CSF, was produced in E. coli as a nonglycosylated form of human G-CSF.

\section{Study design}

Animals were irradiated on day 0 and randomly assigned to a treatment protocol. On day 1 , animals received either $\operatorname{rMGDF}(25 \mu \mathrm{g} / \mathrm{kg} / \mathrm{d}, n=4)$, PEG-rMGDF $(2.5 \mu \mathrm{g} / \mathrm{kg} / \mathrm{d}, n=4)$, r-metHuG-CSF $(10 \mu \mathrm{g} / \mathrm{kg} / \mathrm{d}, n=$ 4), PEG-rMGDF $(2.5 \mu \mathrm{g} / \mathrm{kg} / \mathrm{d})$ and r-metHuG-CSF $(10 \mu \mathrm{g} / \mathrm{kg} / \mathrm{d}, n=$ 4), or HSA (Plasbumin-5 ${ }^{\circledR}$; Miles Inc., Cutter Biological, Elkhart, IN) $(15 \mu \mathrm{g} / \mathrm{kg} / \mathrm{d}, n=13)$. The cytokines or control protein was administered once daily as a 1-ml bolus subcutaneous injection for 18 consecutive days. The level of endotoxin in rMGDF was undetectable $(<0.06$ endotoxin units/mg; Limulus amebocyte lysate assay [Associates of Cape Cod, Inc., Woods Hole, MA]). HSA was not assayed for endotoxin content; however, pyrogenicity tests performed were negative (Miles Pharmaceutical Division, Biological Products, Elkhart, IN).

\section{Clinical support}

Clinical support consisting of an antimicrobial regimen and whole blood transfusions were provided to all animals as required by previously described criteria (30). Fresh, irradiated $\left(1,500 \mathrm{cGy},{ }^{60} \mathrm{Co}\right.$ gamma) packed red blood cells ( $\sim 12.5 \mathrm{ml} /$ transfusion) were administered when the platelet count (PLT) was within normal limits and the hematocrit was $<15 \%$.

\section{Hematologic evaluations}

Peripheral blood. Peripheral blood was obtained from the saphenous vein to assay complete blood (Baker, System 9000; Serono-Baker, Allentown, PA) and differential counts (Wright-Giemsa stain; automated slide stainer, Elkhart, IN). Assessment of hematologic evaluations has been described previously (30).

Bone marrow. Approximately $2 \mathrm{ml}$ of heparinized bone marrow (BM) was aspirated from the humerus and/or iliac crest of anesthetized primates (ketamine, $10 \mathrm{mg} / \mathrm{kg}$ intramuscularly). Low density $\left(<1.077\right.$ grams $\left./ \mathrm{cm}^{3}\right)$ mononuclear cells $(\mathrm{MNC})$ were separated and cultured as previously described (30). GM-CSF and burst-forming units-erythroid (BFU-e)-derived colonies ( $>50$ cells) were ex-

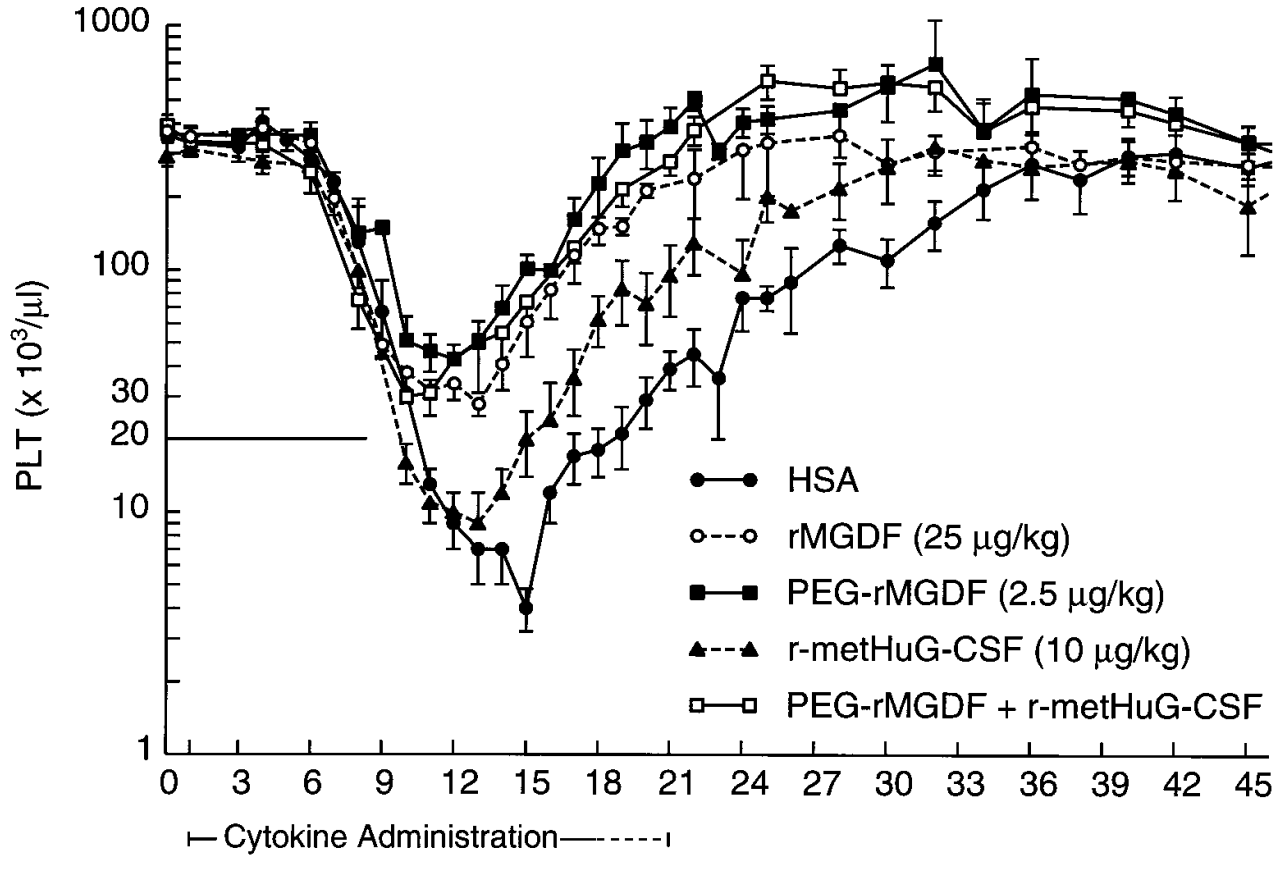

Time (day) Post Irradiation
Figure 1. Effects of cytokine administration on peripheral blood PLT in irradiated primates. The PLT observed in irradiated rhesus primates after rMGDF, PEG-rMGDF, r-metHuG-CSF, PEG-rMGDF combined with r-metHuG-CSF, or HSA administration which were administered as described in Methods. Data represent mean \pm SEM of the absolute PLT for the cytokine or HSA-treated animals. 
Table I. Thrombocytopenia and Neutropenia in Sublethally Irradiated and Cytokine-treated Rhesus Monkeys: Duration, Nadir, and Time to Recovery

\begin{tabular}{|c|c|c|c|c|c|c|}
\hline & \multicolumn{2}{|c|}{ Duration (d) } & \multicolumn{2}{|c|}{$\operatorname{Nadir}\left(\mu \mathrm{l}^{-1}\right)$} & \multicolumn{2}{|c|}{$\begin{array}{l}\text { Time to } \\
\text { recovery }(\mathrm{d})\end{array}$} \\
\hline & THROM & NEUT & Platelet & ANC & Platelet & ANC \\
\hline HSA & 12.2 & 15.5 & 4000 & 2 & 40 & 24 \\
\hline r-metHuG-CSF & $6.7 *$ & $12.3 *$ & $9000 *$ & 11 & 28 & 18 \\
\hline rMGDF & $0.25^{*}$ & $12.3 *$ & $28000 *$ & $40 *$ & 22 & 18 \\
\hline PEG-rMGDF & $0.0^{*}$ & 13.3 & $43000 *$ & $38 *$ & 19 & 17 \\
\hline $\begin{array}{l}\text { PEG-rMGDF + } \\
\text { r-metHuG-CSF }\end{array}$ & $0.5^{*}$ & $9.0 * \neq$ & $30000^{*}$ & $58 * \neq$ & 21 & 17 \\
\hline
\end{tabular}

Monkeys were whole body irradiated to $700 \mathrm{cGy}$ with ${ }^{60} \mathrm{Co}$ gamma radiation and treated with control protein (HSA), r-MGDF, or PEGrMGDF and/or r-metHuG-CSF according to protocol. Neutropenia $(N E U T)$ is defined as ANC $<500 / \mu l$ and thrombocytopenia (THROM) is defined as PLT $<20,000 / \mu \mathrm{l}$. *Statistically different than HSA-treated controls $(P<0.05)$. ${ }^{\ddagger}$ Statistically different than $\mathrm{r}$-metHuG-CSF-treated animals $(P<0.05)$. Days to recovery to baseline were not statistically evaluated.

pressed as the number of CFU/10 $0^{5}$ MNC. MEG-CFU (10-50 cells/colony) are not distinguished from MEG-BFU in this study.

\section{Statistical analysis}

The normal scores test was used to make pair-wise comparisons of the durations of neutropenia and thrombocytopenia and evaluate the statistical significance between nadirs. The tests were carried out using the software package StatXact (Cytel Software Corp., Cambridge, MA). The exact $P$ values were obtained for both analyses. BMderived clonogenic activities were analyzed by two methods. A repeated measures analysis was done followed by a two-sided Dunnett's test to compare treatment days after TBI to baseline values. The comparisons of treated groups versus the time-matched, HSAtreated controls were made using a one-way ANOVA followed by a one-sided Dunnett's test. Clonogenic data were only obtained for two animals administered r-metHuG-CSF, hence statistical analysis was not performed on this data set. These tests were carried out using the software package SYSTAT (SYSTAT, Inc., Evanston, IL).

\section{Results}

Platelet recovery. The administration of rMGDF, PEG-rMGDF, or PEG-rMGDF combined with r-metHuG-CSF significantly enhanced the platelet response in nonhuman primates after TBI. The control-treatment cohort experienced a mean duration of thrombocytopenia (PLT $<20,000 / \mu l$ ) of $12.2 \mathrm{~d}$ which was reduced to 0.25 or $0 \mathrm{~d}$ after rMGDF or PEG-rMGDF administration, respectively $(P<0.001)$ (Fig. 1 , Table I). Similarly, the concomitant administration of PEG-rMGDF and r-metHuG-CSF also reduced the mean duration of thrombocytopenia to $0.5 \mathrm{~d}(P<0.001)$ (Fig. 1, Table I). The r-metHuG-CSF-treated animals experienced a mean duration of thrombocytopenia of $6.7 \mathrm{~d}(P=0.002)$ (Fig. 1, Table I). The mean PLT nadirs in all cytokine-treated groups were significantly improved as compared with the HSA-treated controls $(4,000 / \mu \mathrm{l})$; rMGDF was $28,000 / \mu \mathrm{l}(P=0.003)$, PEGrMGDF alone was $43,000 / \mu l(P=0.003)$, r-metHuG-CSF alone was $9,000 / \mu l(P=0.047)$, and PEG-rMGDF plus

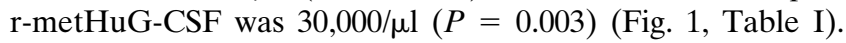
Recovery to baseline PLT levels after TBI occurred earlier in all cytokine-treated groups (rMGDF on day 22, PEG-rMGDF on day 19, r-metHuG-CSF on day 28, PEG-rMGDF plus r-metHuG-CSF on day 21) versus the HSA-treated controls (day 40) (Fig. 1, Table I).

Neutrophil recovery. Neutrophil parameters were also significantly modulated by the above mentioned cytokine protocols (Fig. 2, Table I). The mean duration of neutropenia (absolute neutrophil count $[\mathrm{ANC}]<500 / \mu \mathrm{l})$ was $15.5 \mathrm{~d}$ in the control-treated animals. The administration of r-metHuG-CSF significantly decreased the mean duration of neutropenia to $12.3 \mathrm{~d}(P=0.005)$ (Fig. 2, Table I). Although rMGDF significantly reduced the duration of neutropenia to $12.3 \mathrm{~d}(P=$ $0.014)$, no significant modulation of the duration of neutropenia was observed in the cohort receiving only PEG-rMGDF

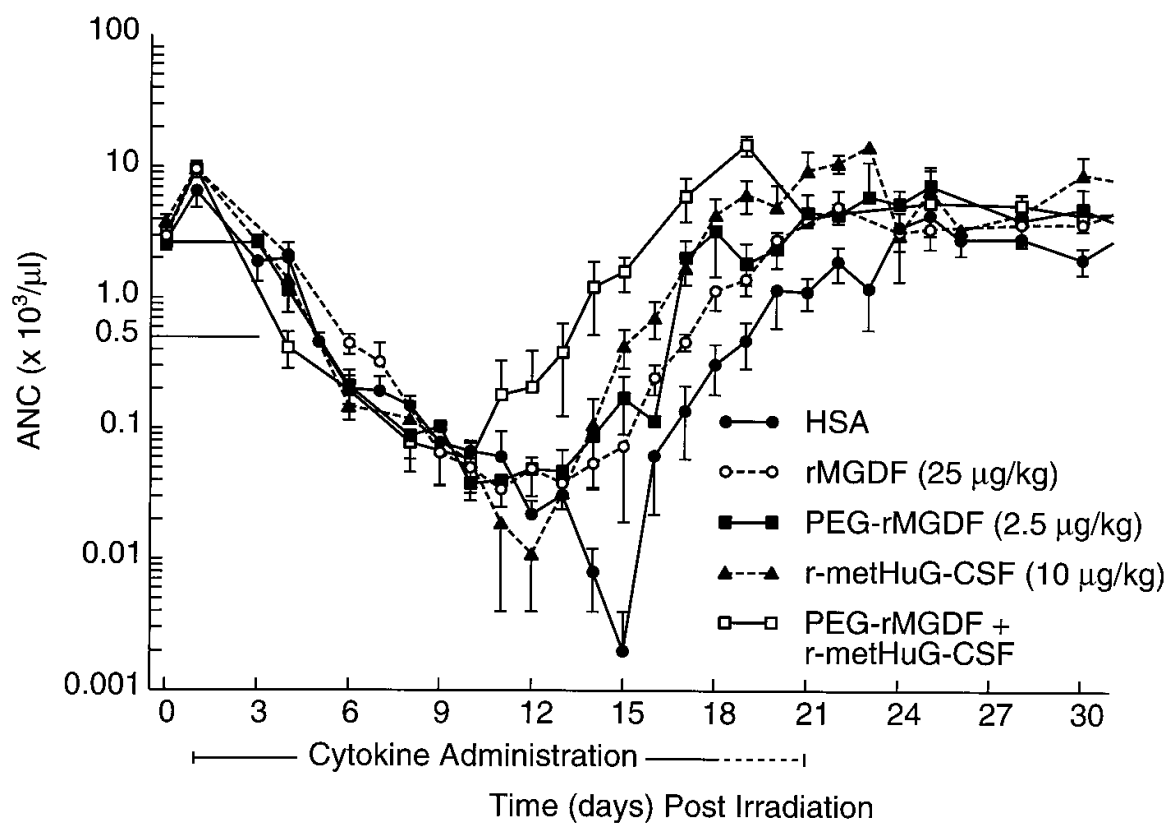

Figure 2. Effects of cytokine administration on peripheral blood ANC in irradiated primates. The ANC observed in irradiated rhesus primates after rMGDF, PEG-rMGDF, r-metHuG-CSF, PEGrMGDF combined with r-metHuG-CSF, or HSA administration which were administered as described in Methods. Data represent mean \pm SEM of the ANC for the cytokine or HSA-treated animals. 
A

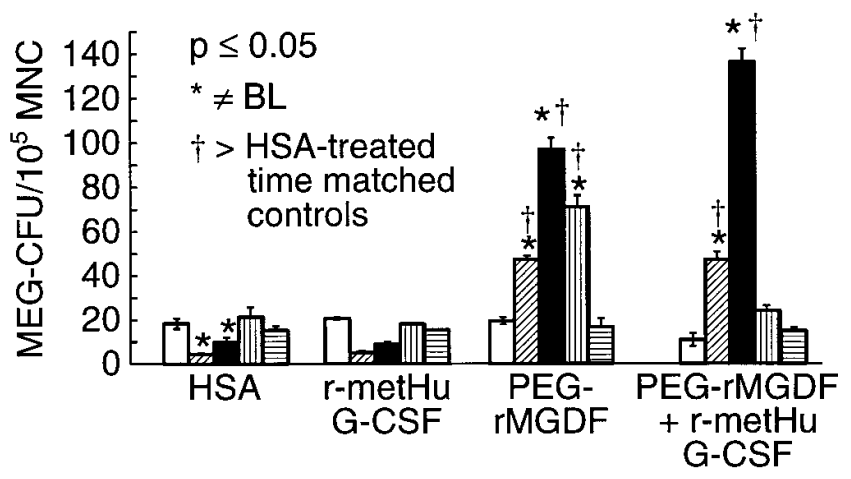

C

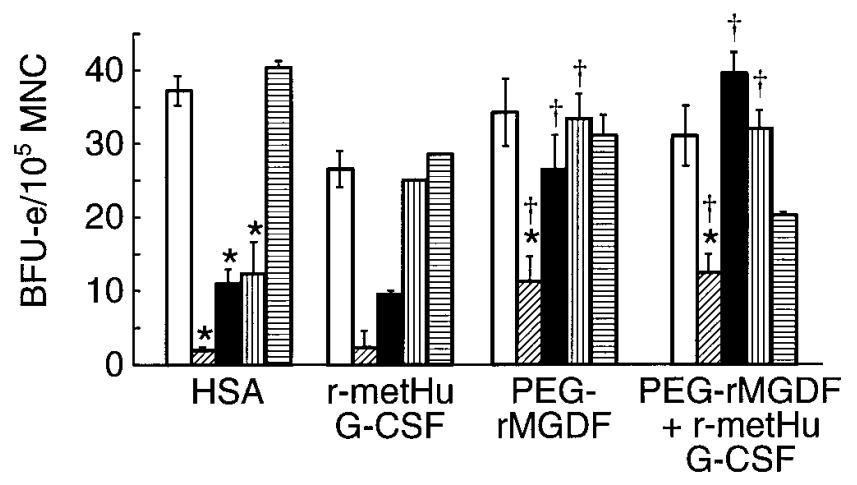

B

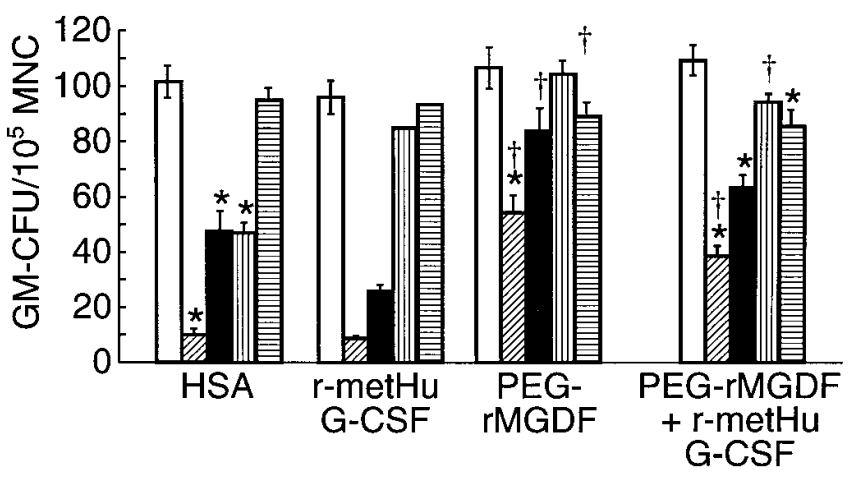

D

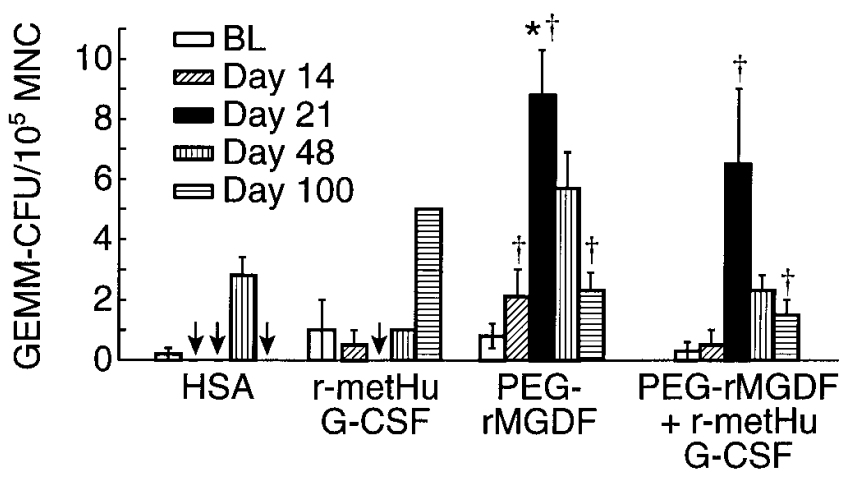

Figure 3. BM-derived concentration of MEG-CFU, GM-CFU, BFU-e, and GEMM-CFUs in irradiated rhesus primates. The $(A) \mathrm{MEG}-\mathrm{CFU},(B)$ GM-CFU, $(C)$ BFU-e, and $(D)$ GEMM-CFU concentrations observed in irradiated rhesus primates before and on days 14, 21, 48, and 100 after TBI and administration with PEG-rMGDF, r-metHuG-CSF, PEG-rMGDF combined with r-metHuG-CSF, or HSA as described in Methods. Clonogenic concentrations are reported as the mean \pm SEM of the CFU per $10^{5}$ MNC BM cells. $B L$, Baseline; $\downarrow$, 0 ; *Significantly different from baseline $(P<0.05)$; ${ }^{\dagger}$ Significantly greater than HSA-treated time-matched controls $(P<0.05)$.

(13.3 d, $P=0.069)$ (Fig. 2, Table I). However, the concomitant administration of the PEG-rMGDF and r-metHuG-CSF produced an additive effect, reducing the mean duration of neutropenia to $9.9 \mathrm{~d}$ which was significantly less than both the HSA-treated controls $(15.5 \mathrm{~d}, P=0.001)$ and the r-metHuGCSF-treated cohort (12.3 d, $P=0.014$ ) (Fig. 2, Table I). The control-treated animals experienced an ANC mean nadir of 2/ $\mu l$. Although the administration of r-metHuG-CSF did not significantly modulate the ANC mean nadir $(11 / \mu 1, P=0.152)$, the administration of rMGDF, PEG-rMGDF, and PEGrMGDF combined with r-metHuG-CSF did significantly improve the mean ANC nadir to $40 / \mu l(P=0.003), 38 / \mu l(P=$ $0.003)$, and $58 / \mu \mathrm{l}(P=0.006)$, respectively (Fig. 2, Table I). The recovery of the ANC to within baseline levels was enhanced by cytokine administration (day 24 in the HSA-treated controls as compared with day 17 in the PEG-rMGDF-treated groups and day 18 in the rMGDF or r-metHuG-CSF-treated groups (Fig. 2, Table I).

$B M$-derived clonogenic activity. BM-derived GM-CFU, BFU-e, MEG-CFU, and granulocyte erythroid megakaryocyte macrophage (GEMM)-CFU activity was evaluated before and at days 14, 21, 48, and 100 after TBI. In the HSA-treated controls the concentration of MEG-CFU was significantly decreased through day 24 and the GM-CFU and BFU-e were significantly decreased through day 48 after TBI (Fig. 3, $A-C$ ).
The number of GEMM-CFU was very low $\left(\leq 1 / 10^{5} \mathrm{MNC}\right)$ in the preirradiated animals and an increase in GEMM-CFU activity for the control group was only observed on day 48 after TBI (Fig. 3 D). Radiation exposure did not statistically decrease the concentration of GEMM-CFU or MEG-CFU in the rMGDF-treated group, whereas the concentration of GMCFU and BFU-e were significantly depressed at days 14 and 28 and recovered to baseline values by day 48 after exposure (Fig. 4, $A-D$ ). The clonogenic activity observed in the PEGrMGDF group was significantly greater than that elicited by rMGDF treatment by day 14 for MEG-CFU, BFU-e, and day 21 for GM-CFU and GEMM-CFU (Fig. 4, $A-D$ ). In contrast to the clonogenic activity observed in animals treated with rMGDF, the administration of PEG-rMGDF also resulted in significantly increased MEG-CFU, GM-CFU, and BFU-e activity above the time-matched HSA controls through day 48 after TBI, as well as a significant increase in GEMM-CFU activity on days 14 and 21 after TBI (Fig. $3, A-D$ ). The combined administration of PEG-rMGDF and r-metHuG-CSF also elicited significant increases above time-matched HSA-treated controls in MEG-CFU (days 14 and 21), GM-CFU (days 14, 21, and 48), BFU-e (days 14, 21, and 48), and GEMM-CFU after TBI (day 21) (Fig. 3, $A-D$ ). Contrary to the HSA treatment, administration of either PEG-rMGDF or PEG-rMGDF and $\mathrm{r}-\mathrm{metHuG}-\mathrm{CSF}$ resulted in MEG-CFU numbers signifi- 

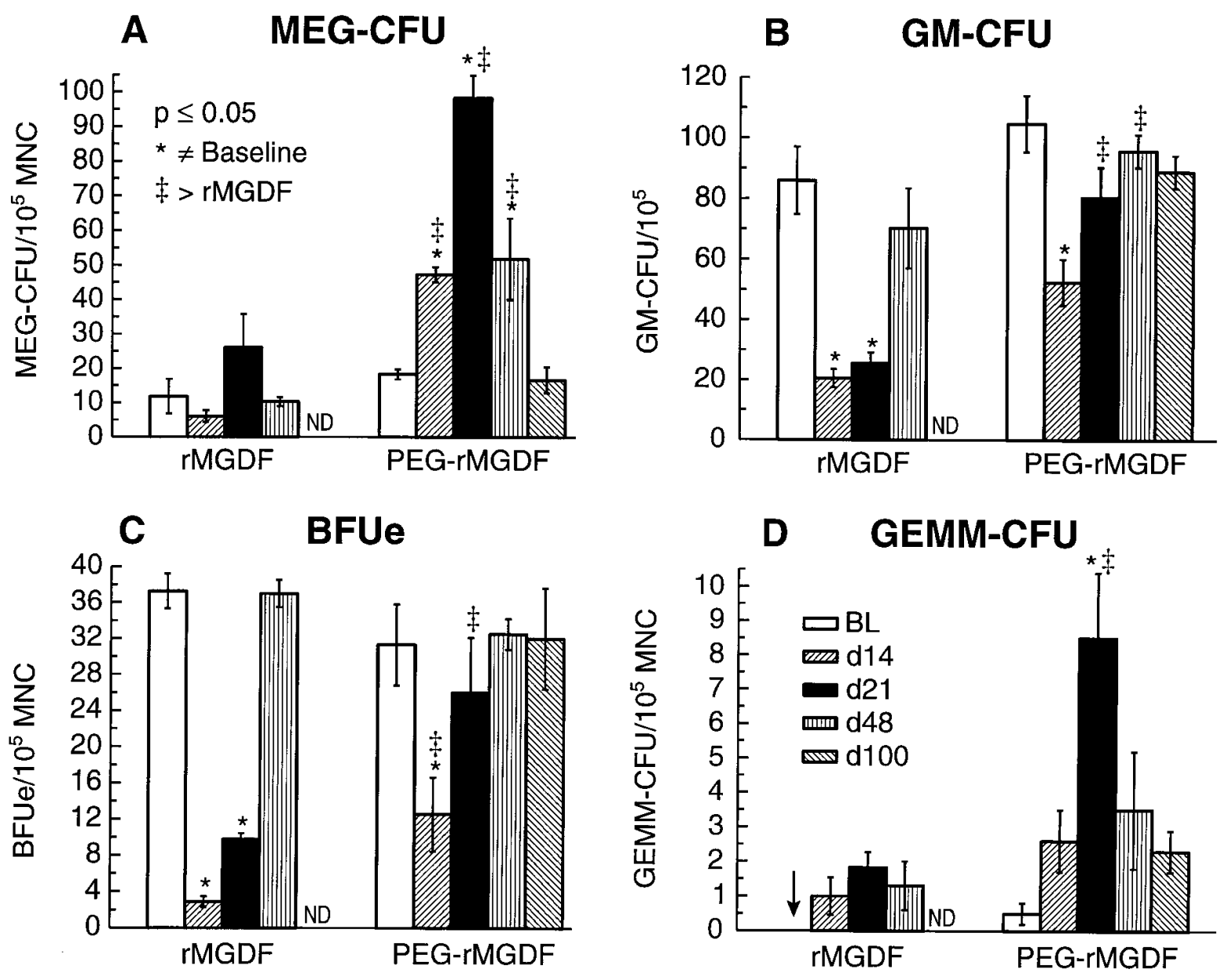

Figure 4. BM-derived concentration of MEG-CFU, GM-CFU, BFU-e, and GEMM-CFUs in irradiated rhesus primates. The $(A)$ MEG-CFU, $(B)$ GM-CFU, $(C)$ BFU-e, and $(D)$ GEMM-CFU concentrations observed in irradiated rhesus primates before and on days 14, 21, 48, and 100 after TBI and administration with rMGDF or PEG-rMGDF as described in Methods. Clonogenic concentrations are reported as the mean \pm SEM of the CFU per $10^{5} \mathrm{MNC}$ BM cells. $B L$, Baseline; $\downarrow, 0 ; N D$, Not done; *Significantly different from baseline $(P<0.05)$; ${ }^{\sharp}$ Significantly greater than rMGDF time-matched controls $(P<0.05)$.

cantly greater than baseline levels on days 14 and 21 after TBI (Fig. $3 A$ ).

\section{Discussion}

In this study, we show that the therapeutic administration of a native or pegylated form of $E$. coli-derived rMGDF can significantly improve platelet nadir, duration of thrombocytopenia, and enhance time to recovery of platelets to normal levels (the three key parameters indicative of increased thrombopoiesis after radiation-induced BM suppression). Furthermore the significantly increased recovery in the marrow-derived MEGCFU activity supports the concept that the enhanced recovery of platelets noted herein is the consequence of the proposed dual action of rMGDF as a stimulator of both megakaryocyte production and maturation. MEG-CFU activity in the PEGrMGDF-treated cohort was significantly increased above baseline values as early as day 14 after TBI, in striking contrast to the significantly depressed activity of the HSA-treated controls.

Increased thrombopoietic efficacy of the PEG-rMGDF molecule has been demonstrated recently in normal and myelosuppressed rodents (22). We have noted a similar greater biologic activity (10-fold) in normal nonhuman primates (Farese, A.M., et al., unpublished data) and confirm herein the increased efficacy of PEG-rMGDF relative to native rMGDF for enhancing hematopoietic recovery in irradiated nonhuman primates. This may be attributed to the significantly increased serum half-life and in vitro biological activity of the pegylated molecule (Cheung, E., et al., unpublished data).

The enhanced recovery of granulopoiesis and stimulation of multilineage BM-derived clonogenic activity was unexpected in light of the lineage dominance shown by the c-mpl ligand in vitro and in vivo in normal animals (9-22, 28, 31-34). Granulopoiesis was stimulated by administration of either the native or the PEG-rMGDF, although the dose of native rMGDF was a log higher than that of the PEG-rMGDF, 25 vs. $2.5 \mu \mathrm{g} / \mathrm{kg}$, respectively. Native rMGDF significantly decreased the duration of neutropenia and both preparations significantly improved the neutrophil nadir, as well as enhanced neutrophil recovery to preirradiation levels. Multilineage hematopoietic recovery was also demonstrated in the clonogenic assays. The BM-derived GM-CFU, BFU-e, and GEMM-CFU activity in the PEG-rMGDF-treated group returned to within or were greater than preirradiation levels by day 21 after TBI. This multilineage effect may be explained by the recent dem- 
onstration that several cytokines can be synthesized constitutively or induced in human marrow-derived megakaryocytes, thus supporting the concept that autocrine and paracrine mechanisms may influence thrombopoiesis as well as proliferation and maturation in other cell lineages. Marrow-derived megakaryocytes are capable of spontaneously secreting IL-3, IL-6, GM-CSF, and IL-1 (35-37). Wickenhauser et al. (36) further demonstrated that IL-3 could significantly enhance the secretion of IL-3, IL-6, and GM-CSF from such marrow-derived megakaryocytes.

It is probable that the enhanced recovery of platelets observed herein is the consequence of the proposed dual action of rMGDF on both stimulation of megakaryocyte progenitors and megakaryocyte endoreduplication and maturation in the postirradiation microenvironment. These activities plus granulopoiesis can be further enhanced through the combined influence of endogenously produced IL-3, IL-6, GM-CSF, IL-11, c-kit ligand, and plasma-derived erythropoietin $(15,18)$. Recent in vitro data have shown enhanced MEG-CFU numbers and/or size in the presence of MGDF or Tpo and additional cytokines $(18,19)$. It is natural to envision from these in vitro experiments the possibility that rMGDF or Tpo may indirectly augment the in situ cytokine milieu by increasing the number of megakaryocytes, which are capable of producing other hematopoietic cytokines, resulting in multilineage stimulation. Although the effects of rMGDF in normal animals appear to be lineage restrictive, the irradiated $\mathrm{BM}$ may provide a unique microenvironment from which progenitor cells renew, differentiate, and mature.

Several studies have evaluated the coadministration of lineage-specific cytokines in the attempt to stimulate multilineage regeneration after BM aplasia. The concurrent administration of IL-3/GM-CSF, as well as PIXY321, the IL-3/GM-CSF fusion protein, was shown to be efficacious in the regeneration of both neutrophils and platelets in nonhuman primates after radiation or drug-induced myelosuppression (38-40). IL-3 and IL-6 and IL-6 and G-CSF, as well as IL-6 and GM-CSF, combination protocols have also shown enhanced neutrophil and platelet recovery relative to controls (41-44) in these nonhuman primate models.

The goal to further enhance the recovery of both neutrophils and platelets after BM aplasia prompted us to evaluate the combined administration of MGDF and r-metHuG-CSF after TBI. Because of the relative efficacy of the pegylated versus the native rMGDF, PEG-rMGDF was used in the combination study. The concomitant administration of PEG-rMGDF and $\mathrm{r}$-metHuG-CSF was equally effective in enhancing platelet recovery versus PEG-rMGDF alone and produced an additive response in neutrophil recovery versus the administration of $\mathrm{r}$-metHuG-CSF alone. The BM-derived clonogenic activity in the group receiving combined PEG-rMGDF and r-met$\mathrm{HuG}-\mathrm{CSF}$ was similar in response to the PEG-rMGDFtreated cohort.

The demonstrated efficacy of rMGDF in significantly improving the thrombocytopenic nadir and decreasing the duration, as well as the time to recovery of circulating platelets after high-dose, sublethal irradiation in the nonhuman primate, forecasts clinical efficacy of this cytokine for treatment for thromobocytopenia in the myelosuppressed patient. We have demonstrated that with the administration of a log lower protein concentration PEG-rMGDF improved all three parameters indicative of platelet recovery after TBI relative to native
rMGDF. In addition, concomitant administration of the lineage-dominant cytokines, PEG-rMGDF and r-metHuG-CSF, stimulated multilineage hematopoietic regeneration in this nonhuman primate model of BM aplasia without apparent competitive decreases in either the granulocyte or megakaryocyte lineage.

\section{Acknowledgments}

The authors wish to thank David Matlick, Michael Flynn, and Nelson Fleming for their superb technical assistance and William Jackson for assistance with the statistical analysis.

\section{References}

1. Kobayashi, Y., T. Okabe, A. Urabe, N. Suzukci, and F. Takaku. 1987. Human granulocyte colony stimulating factor produced by Escherichia coli shortens the period of granulocytopenia induced by irradiation in mice. Jpn. J. Cancer Res. 78:763-768.

2. MacVittie, T.J., R.L. Monroy, M.L. Patchen, and L.M. Souza. 1990. Therapeutic use of recombinant human G-CSF in a canine model of sublethal and lethal whole-body irradiation. Int. J. Radiat. Biol. 57:723-736.

3. Schuening, F.G., R. Storb, S. Goehle, T.C. Graham, F.R. Appelbaum, R. Hackman, and L.M. Souza. 1989. Effect of recombinant human granulocyte colony-stimulating factor on hematopoiesis of normal dogs and on hematopoietic recovery after otherwise lethal total body irradiation. Blood. 74:1308-1313.

4. Patchen, M.L., T.J. MacVittie, B.D. Solberg, and L.M. Souza. 1990. Therapeutic administration of recombinant human granulocyte colony stimulating factor accelerated hemopoietic regeneration and enhances survival in a murine model of radiation-induced myelosuppression. Int. J. Cell Cloning. 8:107-122.

5. Fushiki, M., K. Ono, K. Sasai, Y. Shitamoto, K. Tsutsui, T. Nishidai, M. Takahashi, and M. Abe. 1990. Effect of recombinant human granulocyte colony stimulating factor on granulocytopenia in mice induced by irradiation. Int. J. Radiat. Oncol. Biol. Phys. 18:353-357.

6. Welte, K., M.A. Bonilla, A.P. Gillio, T.C. Boone, G.K. Potter, J.L. Gabrilove, M.A.S. Moore, R.J. O'Reilly, and L.M. Souza. 1987. Recombinant human granulocyte colony-stimulating factor. Effects on hematopoiesis in normal and cyclophosphamide-treated primates. J. Exp. Med. 165:941-948.

7. Morstyn, G., L. Campbell, L.M. Souza, N.K. Alton, J. Keech, M. Green, W. Sheridan, D. Metcalf, and R. Fox. 1988. Effect of granulocyte colony stimulating factor on neutropenia induced by cytotoxic chemotherapy. Lancet. 1:668672

8. Sheridan, W.P., G. Morstyn, M. Wolf, A. Dodds, J. Lusk, D. Maher, J.E. Layton, M.D. Green, L. Souza, and R.M. Fox. 1989. Granulocyte colony-stimulating factor and neutrophil recovery after high-dose chemotherapy and autologous bone marrow transplantation. Lancet. 2:891-895.

9. Bartley, T., J. Bogenberger, P. Hunt, Y.S. Li, H.S. Lu, F. Martin, M.S. Change, B. Samal, J.L. Nichol, S. Swift, et al. 1994. Identification and cloning of a megakaryocyte growth and development factor that is a ligand for the cytokine receptor Mpl. Cell. 77:1117-1124.

10. Hunt, P., Y.S. Li, J.L. Nichol, M.M. Hokom, J.M. Bogenberger, S.E. Swift, J.D. Skrine, A.C. Hornkohl, H. Lu, C. Clogston, et al. 1995. Purification and biological characterization of plasma-derived megakaryocyte growth and development factor. Blood. 86:540-547.

11. Lok, S., K. Kaushansky, R.D. Holly, J.L. Kuljper, C.E. Lofton-Day, P.J. Oort, F.J. Grant, M.D. Heipel, S.K. Burkhead, J.W. Kramer, et al. 1994. Cloning and expression of murine thrombopoietin cDNA and stimulation of platelet production in vivo. Nature (Lond.). 369:565-568.

12. Kato, T., K. Ogami, Y. Shimada, A. Iwamatsu, Y. Schma, H. Akahori, K. Horie, A. Kokubo, Y. Kudo, E. Maeda, et al. 1995. Purification and characterization of thrombopoietin. J. Biochem. 118:229-236.

13. Kuter, D.J., D.L. Beeler, and R.D. Rosenberg. 1994. The purification of megapoietin: a physiological regulator of megakaryocyte growth and development production. Proc. Natl. Acad. Sci. USA. 91:11104-11108.

14. de Sauvage, F.J., P.E. Hass, S.D. Spencer, B.E. Malloy, A.L. Gurney, S.A. Spencer, W.C. Darbonne, W.J. Henzel, S.C. Wong, W.J. Kaung, et al. 1994. Stimulation of megakaryocytopoiesis and thrombopoiesis by the c-Mpl ligand. Nature (Lond.). 369:533-538.

15. Kaushansky, K., S. Lok, R.D. Holly, V.C. Broudy, N. Lin, M.C. Bailey, J.W. Forstrom, M.M. Buddle, P.J. Oort, F.S. Hagen, et al. 1994. Promotion of megakaryocyte progenitor expansion and differentiation by the c-Mpl ligand thrombopoietin. Nature (Lond.). 369:568-571.

16. Wendling, F., E. Maraskovsky, N. Debili, C. Florindo, M. Teepe, M. Titeux, N. Methia, J. Breton-Gorius, D. Cosman, and W. Vainchenker. 1994. c-Mpl ligand is a humoral regulator of megakaryocytopoiesis. Nature (Lond.). 369:571-574. 
17. Zeigler, F.C., F. de Sauvage, H.R. Widmer, G.A. Keller, C. Donahue, R.D. Schreiber, B. Malloy, P. Hass, D. Eaton, and W. Matthews. 1994. In vitro megakaryocytopoietic and thrombopoietic activity of c-mpl ligand (TPO) on purified murine hematopoietic stem cells. Blood. 84:4045-4052.

18. Broudy, V.C., N.L. Lin, and K. Kaushansky. 1995. Thrombopoietin (c-mpl ligand) acts synergistically with erythropoietin, stem cell factor, and interleukin-11 to enhance murine megakaryocyte colony growth and increases megakaryocyte ploidy in vitro. Blood. 85:1719-1726.

19. Nichol, J.L., M.M. Hokom, A. Hornkohl, W.P. Sheridan, H. Ohashi, T. Kato, Y.-S. Li, T.D. Bartley, E. Choi, J. Bogenberger, et al. 1995. Recombinant human megakaryocyte growth and development factor (r-HuMGDF): a cytokine with dual roles in megakaryopoiesis. J. Clin. Invest. 95:2973-2978.

20. Choi, E., M.M. Hokom, T.D. Bartley, Y.S. Li, H. Ohashi, T. Kato, J.L. Nichol, J.D. Skrine, A. Knudten, J. Chen, et al. 1995. Megakaryocyte growth and development factor produces functional human platelets in vitro. Stem Cells. 13:317-321.

21. Ulich, T.R., J. del Castillo, S. Yin, S. Swift, D. Padilla, G. Senaldi, L. Bennett, J. Shutter, J. Bogenberger, D. Sun, et al. 1995. Megakaryocyte growth and development factor ameliorates carboplatin-induced thrombocytopenia in mice. Blood. 86:971-976.

22. Hokom, M.M., D. Lacey, O. Kinsler, E. Choi, S. Kaufman, J. Faust, C. Rowen, E. Dywer, J.L. Nichol, T. Grasel, et al. 1995. Megakaryocyte growth and development factor abrogates the lethal thrombocytopenia associated with carboplatin and irradiation in mice. Blood. 86:4486-4492.

23. Kindler, V., B. Thorens, S. de Kossodo, B. Allet, J.F. Eliason, D. Thatcher, N. Farber, and P. Vassalli. 1986. Stimulation of hematopoiesis in vivo by recombinant bacterial murine interleukin 3. Proc. Natl. Acad. Sci. USA. 83: 1001-1005.

24. Metcalf, D., C.G. Begley, G.R. Johnson, N.A. Nicola, A.F. Lopez, and D.J. Williamsen. 1986. Effects of purified bacterially synthesized murine multiCSF (IL-3) on hematopoiesis in normal adult mice. Blood. 68:46-57.

25. Ishibashi, T., H. Kimura, Y. Shikama, T. Uchida, S. Kariyone, T. Hirano, T. Kishimoto, F. Takatsuki, and Y. Akiyama. 1989. Interleukin-6 is a potent thrombopoietic factor in vivo in mice. Blood. 74:1241-1244.

26. Metcalf, D., N.A. Nicola, and D.P. Gearing. 1990. Effects of injected leukemia inhibitory factor (LIF) on hemopoietic and other tissues in mice. Blood. 76:50-56.

27. Neben, T.Y., J. Loebelenz, L. Hayes, K. McCarthy, J. Stoudemire, R. Schaub, and S.J. Goldman. 1993. Recombinant human interleukin-11 stimulates megakaryocytopoiesis and increases peripheral platelets in normal and splenectomized mice. Blood. 81:901-908.

28. Farese, A.M., P. Hunt, T.C. Boone, and T.J. MacVittie. 1995. Recombinant human megakaryocyte growth and development factor (r-HuMGDF) simulates megakaryocytopoiesis in normal primates. Blood. 85:55-59.

29. The Institute of Laboratory Animal Resources, National Research Council. Guide for the Care and Use of Laboratory Animals. Washington, DC, National Institutes of Health. Publication No. 86-23.

30. Farese, A.M., F. Herodin, L.A. Grab, N. Varma, C. Baum, E. Burton, J.P. McKearn, and T.J. MacVittie. 1996. Acceleration of hematopoietic reconstitution with a synthetic cytokine (SC-55494) after radiation-induced marrow aplasia. Blood. 87:581-591.

31. Methia, N., F. Louache, W. Vainchenker, and F. Wendling. 1994. Oli- godeoxynucleotides antisense to the proto-oncogene c- $m p l$ specifically inhibit in vitro megakaryocytopoiesis. Blood. 82:1395-1401.

32. Debili, N., F. Wendling, D. Cosman, M. Titeux, C. Florindo, I. Dusanter-Fouter, K. Schooley, N. Methia, M. Charon, R. Nador, et al. 1995. The $\mathrm{Mpl}$ receptor is expressed in the megakaryocytic lineage from late progenitors to platelets. Blood. 85:391-401.

33. Gurney, A.L., K. Carver-Morre, F.J. de Sauvage, and M.W. Moore. 1994. Thrombocytopenia in c-mpl-deficient mice. Science (Wash. DC). 265: 1445-1447.

34. Yan, X.-Q., D. Lacey, F. Fletcher, C. Hartley, T. McElroy, Y. Sun, B. Shannon, M. Xia, S. Mu, C. Saris, et al. 1995. Chronic exposure to retroviral vector encoded MGDF ( $\mathrm{mpl}$-ligand) induced lineage-specific growth and differentiation of megakaryocytes in mice. Blood. 86:4025-4033.

35. Jiang, S., J.D. Levine, Y. Fu, B. Deng, R. London, J.E. Groopman, and H. Avraham. 1994. Cytokine production by primary bone marrow megakaryocytes. Blood. 84:4151-4156.

36. Wickenhauser, C., J. Lorenzen, J. Thiele, A. Hillienhof, B. Jungheim, M.-L. Hansmann, and R. Fischer. 1995. Secretion of cytokines (interleukins-1 $\alpha$, -3 , and -6 and granulocyte macrophage colony-stimulating factor) by normal human bone marrow megakaryocytes. Blood. 85:685-691.

37. Navarro, S., N. Debili, J.-P. Le Couedic, B. Klein, J. Breton-Gorius, J. Doly, and W. Vainchenker. 1991. Interleukin-6 and its receptor are expressed by human megakaryocytes: in vitro effects on proliferation and endoreplication. Blood. 77:461-471.

38. Farese, A.M., D.E. Williams, F.R. Seiler, and T.J. MacVittie. 1993. Combination protocols of cytokine therapy with interleukin-3 and granulocytemacrophage colony-stimulating factor in a primate model of radiation-induced marrow aplasia. Blood. 82:3012-3018.

39. Williams, D.E., J.T. Dunn, L.S. Park, E.A. Frieden, F.R. Seiler, A. Farese, and T.J. MacVittie. 1993. A GM-CSF/IL-3 fusion protein promotes neutrophil and platelet recovery in sublethally irradiated rhesus monkeys. Bio Therapeutics. 4:17-29.

40. Williams, D.E., A. Farese, and T.J. MacVittie. 1993. PIXY321, but not GM-CSF plus IL-3, promotes hematopoietic reconstitution following lethal irradiation. Blood. 82:366a. (Abstr.)

41. MacVittie, T.J., A.M. Farese, M.L. Patchen, and L.A. Myers. 1994 Therapeutic efficacy of recombinant interleukin- 6 alone and combined with recombinant human IL-3 in a nonhuman primate model of high-dose, sublethal radiation-induced marrow aplasia. Blood. 84:2515-2522.

42. Winton, E.F., J. Srinivasiah, B.K. Kim, C.D. Hillyer, E.A. Storbert, J.L. Orkin, R.B. Swenson, H.M. McClure, L.A. Myers, and R. Saral. 1994. Effect of recombinant human interleukin-6 and rhIL-3 on hematopoietic regeneration as demonstrated in a nonhuman primate chemotherapy model. Blood. 84:65-73.

43. Farese, A.M., L.A. Myers, and T.J. MacVittie. 1994. Therapeutic efficacy of the combined administration of either recombinant human interleukin-6 and rh-granulocyte colony stimulating factor or rh-granulocyte-macrophage colony-stimulating factor in a primate model of radiation-induced marrow aplasia. Exp. Hematol. 22:684a. (Abstr.)

44. Gonter, P.W., C.D. Hillyer, E.A. Strobert, J.L. Orkin, R.B. Swenson, H.M. McClure, L.A. Myers, and E.F. Winton. 1993. Enhanced post-chemotherapy platelet and neutrophil recovery using IL-6 and rhGM-CSF in a nonhuman primate model. Blood. 82(Suppl. 1):365a. (Abstr.) 